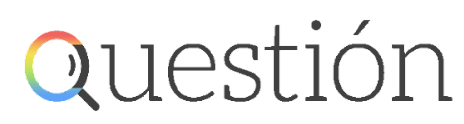

Periodismo / Comunicación ISSN 1669-6581

Enseñar y aprender en contexto de ASPO y encierro punitivo

Natalia Rosana Zapata, Malena García

Question/Cuestión, Nro.70, Vol.3, diciembre 2021

ISSN: 1669-6581

URL de la Revista: https://perio.unlp.edu.ar/ojs/index.php/question/

IICom -FPyCS -UNLP

DOI: https//doi.org/10.24215/16696581e657

\title{
Enseñar y aprender en contexto de ASPO y encierro punitivo
}

Teaching and learning in the context of ASPO and punitive confinement

\author{
Natalia Rosana Zapata \\ UNLP, FPyCS, INESCO
}

Argentina

natalia.zapata@perio.unlp.edu.ar https://orcid.org/0000-0001-6297-9785

Malena García

UNLP, FPyCS, INESCO/CONICET

Argentina

malena garcia@live.com

https://orcid.org/0000-0002-8758-7080 


\section{Resumen}

Presentamos aquí el relato de una experiencia pedagógica atravesada por el contexto de aislamiento social, preventivo y obligatorio (ASPO) durante los ciclos lectivos 2020 y 2021, en la sede Unidad Penitenciaria 9 de La Plata (o Extensión áulica UP9) de la Facultad de Periodismo y Comunicación Social (FPyCS) de la Universidad Nacional de La Plata (UNLP).

Nuestra práctica docente se enmarca en la materia Modernidades, Medios y Poder, cátedra II (MMP II), una asignatura correspondiente al primer tramo del ciclo básico, para la Tecnicatura en Comunicación Social (título intermedio) de la Licenciatura en Comunicación Social; también la cursan estudiantes de la carrera Profesorado en Comunicación Social de la FPyCS-UNLP. En el Plan anterior (1998) la materia se denomina "Comunicación y Medios" y se ubica en el ciclo básico para ambas carreras.

Palabras claves: comunicación, cárceles, pandemia

\section{Abstract}

We present here the account of a pedagogical experience crossed by the context of social, preventive and compulsory isolation (ASPO) during the 2020 and 2021 school years, at the Penitentiary Unit 9 headquarters in La Plata (or UP9 classroom extension) of the Faculty of Journalism and Social Communication (FPyCS) of the National University of La Plata (UNLP).

Our teaching practice is framed in the subject Modernities, Media and Power, chair II (MMP II), a subject corresponding to the first section of the basic cycle, for the Technician in Social Communication (intermediate degree) of the Bachelor's Degree in Social Communication; It is also attended by students of the Professorship in Social Communication at the FPyCS-UNLP. In the previous Plan (1998) the subject is called "Communication and Media" and is located in the basic cycle for both careers.

Keyword: communication, prisons, pandemic, 


\section{Estrategias institucionales ante la emergencia sanitaria}

Presentamos aquí el relato de una experiencia pedagógica atravesada por el contexto de aislamiento social, preventivo y obligatorio (ASPO) durante los ciclos lectivos 2020 y 2021, en la sede Unidad Penitenciaria 9 de La Plata (o Extensión áulica UP9) de la Facultad de Periodismo y Comunicación Social (FPyCS) de la Universidad Nacional de La Plata (UNLP).

Nuestra práctica docente se enmarca en la materia Modernidades, Medios y Poder, cátedra II (MMP II), una asignatura correspondiente al primer tramo del ciclo básico, para la Tecnicatura en Comunicación Social (título intermedio) de la Licenciatura en Comunicación Social; también la cursan estudiantes de la carrera Profesorado en Comunicación Social de todas carreras del Plan de Estudios vigente en la FPyCS-UNLP. En el Plan anterior (1998) la materia se denomina "Comunicación y Medios" y se ubica en el ciclo básico para ambas carreras.

Ante la emergencia sanitaria, a pocos días de iniciar el ciclo lectivo 2020, desde el equipo de cátedra adaptamos los contenidos y materiales a la estrategia de educación a distancia (EAD) y la Facultad nos proporcionó un espacio en Web de cátedras, donde dispusimos el programa, cronograma, bibliografía de clases teóricas y prácticas, videos, entre otros recursos.

Asimismo, establecimos un acuerdo, en el marco del equipo de cátedra, para que cada docente, tanto de las clases teóricas como de las cinco comisiones de las clases prácticas, desarrolle un aula virtual en la plataforma Google Classroom.

El portal "Web de cátedras" forma parte de una estrategia general de la institución, donde la información de las cátedras se encuentra disponible, es posible recorrerla y descargar material de forma ágil y con economía de datos, tras la iniciativa multiactoral para bonificar la navegación por sitios con extensión “.edu.ar" y "unlp.edu.ar” en el marco de la emergencia.

Para el caso que aquí relatamos, la experiencia del dictado de la asignatura en el marco de la Extensión Áulica Unidad 9 de la FPyCS, empleamos durante el ciclo lectivo 2020 la referida web (que permite alojar materiales en el repositorio de la unidad académica), y el correo 
electrónico institucional de la cátedra como herramientas privilegiadas para establecer la interacción entre equipo docente y estudiantes. Asimismo, durante el ciclo lectivo 2021 sumamos el Google Meet para encuentros sincrónicos quincenales.

Cabe destacar que el tránsito de la experiencia docente en contexto de ASPO, estuvo acompañada de un proceso de capacitación, por medio de encuentros de asesorías en línea para la educación a distancia, organizados por el Programa de Apoyo a la Educación a Distancia de la Secretaría Académica de la UNLP. Además, el equipo docente que integra la cátedra, siempre se ha interesado y cuestionado el proceso de subjetivación y las relaciones entre jóvenes y tecnologías de la información y la comunicación. En este sentido, la virtualización completa de la propuesta del curso tuvo un impacto propositivo en el que compartimos experiencias, herramientas y diálogos tendientes a resolver las dificultades.

\section{Extensión Áulica U9: un doble aislamiento}

La FPyCS tiene una vasta trayectoria en acciones de extensión universitaria, con la intención de llevar la Universidad allí donde históricamente nunca antes se había acercado: los territorios que no cuentan con un establecimiento universitario cercano o si cuentan con uno, las personas no habían logrado ingresar para cursar carreras de grado. Uno de esos territorios son los establecimientos carcelarios.

En tal sentido, la Extensión Áulica UP9 se inició en el mes de octubre de 2009, como un dispositivo más en el marco de las políticas de inclusión universitaria para garantizar el legítimo derecho a la educación, en este caso de las personas privadas de libertad, garantizado en la Constitución Nacional y especificado en el capítulo XII de la Ley de Educación Nacional (26206/06).

Se trata de un espacio propio tras los muros de la Unidad Penitenciaria $\mathrm{N}^{\circ} 9$ localizada en el barrio Villa Elvira de la ciudad La Plata, en donde se desarrollan las cursadas correspondientes 
al ciclo básico y la Facultad facilita una oferta de comisiones de materias del ciclo superior de las carreras Licenciatura y Profesorado en Comunicación Social.

En la Extensión UP9 cursan personas privadas de su libertad alojadas en la UP 9, así como también en otras unidades carcelarias pertenecientes al Servicio Penitenciario Bonaerense (SPB), tanto "femeninas" (UP 8 y UP 33) como "masculinas" (UP 1 Olmos, UP 12 y 18 Gorina; UP 24 y UP31 Florencio Varela, entre otras). Las y los estudiantes son trasladados a dichas instalaciones para poder cursar las asignaturas y generar vínculos con el Centro de Estudiantes Universitarios "Santo Tomás de Aquino" (CEUSTA), espacio autogestivo que nuclea a estudiantes de distintas carreras de la UNLP.

A instancias de una resolución del Tribunal de Casación Penal bonaerense, en abril del 2020 el SPB instrumentó el "Protocolo para el uso de teléfonos celulares por parte de personas privadas de la libertad en el ámbito del Servicio Penitenciario Bonaerense", que contempla la posibilidad de que las personas privadas de libertad registren un dispositivo electrónico (teléfono, tablet o notebook) y "legalicen" su uso al interior del penal con fines educativos, culturales y de comunicación familiar.

A partir de esta disposición, las familias pudieron proveer los aparatos, tarjetas con abonos limitados y desde los proyectos de extensión y de las agrupaciones estudiantiles se iniciaron campañas de donación de elementos de higiene y de smartphones destinados a este grupo minoritario de la población carcelaria. Sin embargo, no todas y todos los estudiantes universitarios en contextos de encierro pudieron acceder a un teléfono celular.

En este marco, la comisión de la materia en dicho espacio territorial, también pasó a la virtualidad y se nos presentaron varios desafíos a considerar, teniendo en cuenta aspectos estructurales y coyunturales en el caso de este grupo de estudiantes que cursa en la Sede UP9: las condiciones de detención signadas por la superpoblación, el hacinamiento, la carencia de recursos básicos para garantizar la seguridad alimentaria y sanitaria; la falta de contacto con familiares y sus defensores ante el doble aislamiento social que se encuentran padeciendo en 
medio de la pandemia y el ASPO (las propias personas presas decidieron suspender las visitas de familiares y personas queridas para evitar contagios).

A esto se suma la carencia de una estructura que permita la conectividad a internet y la falta de recursos técnicos y tecnológicos para garantizar la continuidad pedagógica; los protocolos internos del sistema penitenciario que limitan la circulación al interior de las unidades carcelarias ante la amenaza de contagios masivos de coronavirus; y que el estudiantado no puede acceder a los espacios colectivos de estudio como los Centros de Estudiantes y/o Bibliotecas.

\section{Transitar la cursada por celular}

Nuestra materia tiene como objetivo abordar la relación dialéctica entre sociedad moderna, medios de comunicación e industrias culturales a lo largo de la historia. La asignatura está estructurada en tres grandes ejes de contenidos: modernidad/modernidades; medios, comunicación/poder; globalización/convergencia. La cursada está dirigida principalmente a estudiantes ingresantes, personas que por primera vez tienen acercamiento a la vida universitaria. 


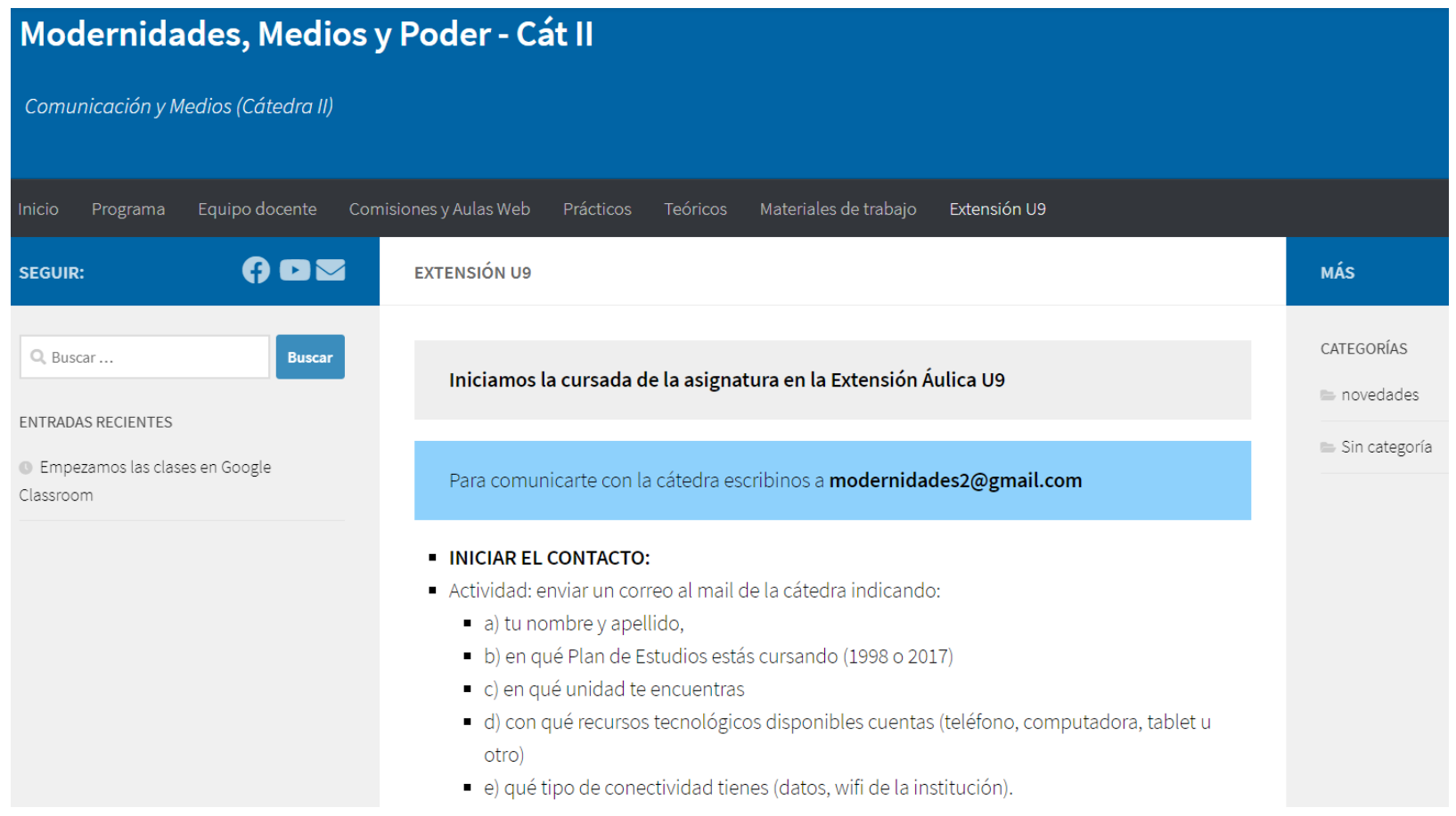

Figura 1: Captura de la web institucional de la materia. Pestaña Extensión áulica U9. (Modernidades, Medios y Poder. Cát II, 2021).

Al diseñar la propuesta pedagógica específica para la Extensión U9, contábamos con un diagnóstico inicial proporcionado por el Programa de Educación Superior en Cárceles (EduCa) de la Facultad, que nos ilustraba la crítica situación: las y los estudiantes no cuentan con una PC propia desde donde trabajar, no tienen acceso a Internet y, en el mejor de los casos, sólo utilizan los PCs de uso colectivo y/o celulares con datos móviles limitados para acceder al material y realizar los trabajos prácticos y evaluaciones.

Ante este escenario, desde la cátedra decidimos conformar un equipo de docentes para acompañar el proceso pedagógico específico que requiere la Extensión Áulica. Desde este equipo definimos no usar Classroom, diseñar las clases escritas y facilitar el material bibliográfico, ambos recursos en archivos formato pdf, que publicamos en el día y horario pautado para la cursada en la web de cátedra, que tiene extensión ".edu.ar" y posibilita la 
navegación gratuita a partir del compromiso asumido por el Estado y las empresas prestadoras de servicios de telefonía e internet. Generamos una pestaña específica en la web para publicar contenidos destinados a la Extensión U9.

La planificación del recorrido pedagógico estuvo abocada al diseño de diez clases escritas pensadas para el contexto de emergencia, donde las y los estudiantes no eligieron una cursada "a distancia" sino que esta modalidad se definió atendiendo a la coyuntura del contexto, pues en marzo nos esperaban en las aulas de la cárcel. En el diseño de dichas clases tuvimos en cuenta una estructura común: ubicación en el eje temático, introducción, mediación de la bibliografía con inclusión de recursos gráficos (imágenes, mapas, cuadros, viñetas), recursos de interpelación (interrogantes reflexivos), cierre y propuesta de actividad práctica obligatoria con distintos niveles de complejidad (actividades conceptuales, de análisis y/o de producción).

En la propuesta pedagógica contemplamos, asimismo, el recorrido educativo previo del grupo de estudiantes (en su mayoría ingresantes) y seleccionamos del Programa materiales para facilitar la lectura y el desarrollo de tareas prácticas sin afectar la calidad de la propuesta en general, pero siguiendo los criterios de accesibilidad y adaptabilidad.

De cara al inicio del primer cuatrimestre 2020 abandonamos la posibilidad de proponer material audiovisual ya que, en general, dichos materiales se enlazan en sitios externos a los de las universidades nacionales y por ello su visualización consume datos y el estudiantado de la Extensión Áulica no cuenta con créditos suficientes telefónicos garantizados para tal fin. También desistimos de los encuentros sincrónicos mediante videollamada o videoconferencia, justamente para evitar el consumo de datos a través de plataformas externas a la extensión ".edu.ar" (aunque finalmente realizamos un encuentro por plataforma Zoom, a pedido del grupo de estudiantes de cara a la evaluación integradora).

No obstante, en el siguiente ciclo lectivo, teniendo diagnósticos más certeros sobre la conectividad de cada estudiante, habiéndose generado espacios colectivos reconexión en los centros universitarios de las unidades penitenciarias $\mathrm{y}$, sobre todo por la demanda expresada 
por las y los estudiantes, incorporamos encuentros sincrónicos quincenales además de la web y el correo electrónico.

\section{Enseñar y aprender a la distancia}

Nuestra materia está dirigida, principalmente, a estudiantes ingresantes. En tal sentido, entendemos junto con Claudia Bracchi (2016) que el ingreso a una carrera universitaria implica para la persona una reconfiguración subjetiva en la que "se ponen en juego los esquemas de percepción, de apreciación, valoración y de acción (habitus) que harán posible diversas tomas de decisiones contribuyendo a conformar el oficio de estudiante universitario".

El oficio de estudiante universitario es parte de un proceso de aprendizaje no curricularizado que se conforma, fundamentalmente, cuando una persona logra apropiarse de las reglas del juego propias de la institución universitaria y, en el tránsito por ese camino genera relaciones estratégicas en términos de los saberes y recursos (capitales económicos, culturales y simbólicos) que la persona tiene acumulados y, a su vez, adquiere otros saberes y recursos.

En la virtualización de las clases ese oficio se vio implicado en la apropiación de herramientas digitales: contar con un correo electrónico personal, navegar la web de cátedra, leer desde el dispositivo disponible (fundamentalmente el celular) y realizar los trabajos prácticos adaptando el dispositivo mediante aplicaciones de procesamiento de texto.

En el ciclo lectivo 2020 la primera semana de cursada se contactaron ocho estudiantes (de veintitrés que figuraban en acta de inscripción) al correo electrónico institucional que pusimos a disposición para la interacción; en la segunda semana el nivel de la comunicación decayó y ya en la tercera semana de clases llegaron los primeros trabajos prácticos; la cantidad de estudiantes se mantuvo entre ocho y diez durante toda la cursada. En tanto, al ciclo lectivo 2021 se inscribieron 50 estudiantes, de ese total realizaron el trabajo integrador final de evaluación 28 cursantes.

Cada miércoles, en el horario pautado para el dictado de la materia, enviamos un correo electrónico personalizado con el contenido de cada clase. Durante el proceso pedagógico 
procuramos la interacción permanente a través del mail, no sólo para la recepción y envío de materiales y devolución de las actividades prácticas, sino que también para la generación de un diálogo con intercambio de ideas en torno a esos materiales y/o ejercicios.

A través del mail recibimos los trabajos prácticos y otros mensajes que el estudiantado consideraba hacernos llegar. Asimismo, durante la semana, acercamos por el mismo medio las devoluciones de las actividades prácticas realizadas. Como equipo docente nos dividimos la tarea para realizar el acompañamiento específico de cada estudiante durante todo el proceso de la cursada.

En cuanto a los trabajos prácticos, en algunos casos produjeron las actividades en hoja de papel, sacaron fotos de la producción y enviaron por correo; en otros casos hicieron llegar sus trabajos en documentos de Word o, directamente, mandaron su producción pegada al cuerpo del correo electrónico.

La priorización de contenidos y la elaboración de materiales específicos para consultar en cualquier momento de la semana, permitió que el grupo de estudiantes pueda realizar la cursada asincrónicamente, pudiendo acceder a la clase en distintos momentos, sobre todo para quienes no disponían de dispositivos propios, sino compartidos. Por otro lado, como una limitante significativa en el proceso educativo, la estrategia dificultó el intercambio de reflexiones y dudas sobre los contenidos de la clase, algo que en la presencialidad se da de manera diferente, en particular mediante la socialización, que enriquece los procesos de enseñanza y aprendizaje.

La interacción en línea implica que las reflexiones, las preguntas, esos intercambios propios del espacio áulico, se den de modo particularizado: el o la estudiante nos realiza una consulta por correo electrónico y nosotras, desde el equipo docente, le respondemos; o nos envía su trabajo práctico y le realizamos una devolución. En algunos casos, se generan significativos hilos de diálogo entre estudiantes y equipo docente en esa interacción.

La experiencia del ciclo lectivo 2020 limitó el hecho de aprender de las dudas planteadas por las y los compañeros en clase, así como también el intercambio entre estudiantes al realizar un 
trabajo en conjunto, compartir sus apreciaciones acerca de lo trabajado en el aula o intercambiar acerca de otras cuestiones y transitar las cursadas en colectivo. Sin embargo, tras haber atravesado un año completo de aislamiento social y virtualización de las clases, en el siguiente ciclo académico los encuentros por Google Meet posibilitaron el acercamiento entre estudiantes y docentes, la escucha y la consulta in situ además de darse algunos intercambios.

En consecuencia, lo que Claudia Bracchi (2004) denomina como "el oficio de estudiante", que se construye a partir de articular las actividades de formación y participación en la vida universitaria es absolutamente desafiante en las clases en línea, y más aún, en los contextos de encierro punitivo, donde la presencia de la Universidad no solo tiene el objetivo de acercar materias de grado sino instituirse como un lugar de referencia ante la hostilidad carcelaria, como intermediaria con otros actores y canalizadora de las denuncias ante situaciones de vulneración de derechos, malos tratos y tortura.

Por otra parte, en el contexto de la cárcel, al igual que en el edificio de la Facultad, cuando nos encontramos docentes y estudiantes en una clase presencial, la clase no empieza y termina en el horario pautado, así como tampoco empieza y termina en las cuatro paredes del aula asignada. La recorrida obligada y el saludo por la Biblioteca, acercarnos al Centro de Estudiantes, compartir un mate, recibir un pedido de materiales, de trámites administrativos o académicos, una denuncia... Alguien que nos muestra la huerta, o el mural que está pintando para darle color al Centro Universitario, u otra persona que nos hace saber los problemas eternos para comunicarse con su juzgado o defensa.

\section{Construir el espacio áulico mediante la pantalla}

En cuanto a las consignas para problematizar los ejes de la materia, procuramos estrategias didácticas que permitieran al grupo de estudiantes "saber hacer" con eso que saben y que muchas veces no saben que lo saben, hasta no verlo en acción: sintetizar ideas, tomar 
posición, narrar de diversas y creativas maneras. Lo trabajamos a través de la auto narración, preguntas reflexivas, visionado de vídeos, análisis de noticias, producción de mapas conceptuales, líneas de tiempo y crónicas breves.

Una de las primeras consignas, proponía presentarse, comentar los recursos tecnológicos y de conectividad con los que contaba y, a la vez, construir una definición de comunicación y pensarse como comunicadores:

Para mí la comunicación es la manera que tenemos para relacionarnos unes con los otres. Me gusta la relación contemporánea que se da entre la modernidad, medios y poder...saludos \& $\&$ (Gerardo)

La comunicación es un medio para relacionarse, informar o comunicar a cualquier ámbito social. Lo que me entusiasma de la carrera es poder llegar a todos los ámbitos sociales con información. Apunto a ser periodista deportivo ya que fuí deportista y me interesa el fútbol. (Hernán).

Es una forma de expresar ideas, sentimientos y emociones. Lo que me entusiasma es poder generar en la otra persona un pensamiento o un punto de vista distinto a lo que creía. (Mauricio)

La comunicación para mí es una herramienta social, que permite visualizar y darles voz a determinadas situaciones y/o acontecimientos (...) También creo que muchas veces sirve como método de dominación. (Marcelo) 
Me encuentro en la unidad N 9, mi conectividad es vía celular y veo todos los noticieros especialmente Tele9. Quisiera aprender y poder contar todo lo que pasa en la Universidad en contexto de encierro como la realidad que vivimos los privados de la libertad día a día. (Andrés)

Otra de las consignas buscaba recuperar las experiencias y trayectorias de consumo en relación a los medios de comunicación: qué dibujos animados veían en la infancia, qué revistas y diarios leían, qué programas de televisión, películas y música, así como los accesos a dispositivos tecnológicos. En el proceso de recuperar sus trayectorias, trabajamos en la reflexión como consumidores de medios de comunicación y como sujetos que, en determinadas coberturas, los medios pueden estigmatizar.

En la clase seis propusimos la escritura de un relato, de tres carillas como máximo, donde se relatara la relación con los medios de comunicación, poniendo en contexto histórico cada momento narrado. Aquí compartimos algunos de los fragmentos de ese trabajo:

Mi primer celular fue un Nokia, esos que parecían un ladrillo y que te cubrían la mitad del rostro. Cuando lo empecé a usar era algo increíble y sorprendente ya que podía llamar a mi madre a la distancia, era como tenerla cerca, era maravilloso hablar con las personas o mandar mensajes de texto e investigar para que servía cada cosa que tenía en las herramientas ese aparato blanco y negro (Jannneth)

En 2001 volví a escuchar radio por encontrarme nuevamente en contexto de encierro, había una radio de cumbias que se llamaba "el correo de los privados" y donde llamaban a pedir canciones de diferentes institutos, penales o familiares de chicos que estaban privados de la libertad, siempre la escuchábamos con mis compañeros de pabellón. (Jonathan) 
Hoy estando catorce años detenido (...) al ir redactando este breve resumen de historia personal, me doy cuenta de que soy un afortunado de la vida, estuve alrededor de la muerte varias veces y no lo ví. A su vez, me doy cuenta de que hoy cumplo un sueño que alguna vez tuve, el de cursar una carrera universitaria, que jamás pensé que llegaría a concretar, y es increíble también ver cómo avanzó la tecnología desde que tengo noción, que hoy por medio de un celular puedo recibir la información para poder rendir y cursar estas y otras materia de la carrera. (Juan)

Otro suceso que recuerdo fue un tiroteo en mi barrio Ejército de los Andes, donde quien cubrió la nota periodística fue José de Zer, quien apodó al barrio como "Fuerte Apache" generando el estigma de que ahí adentro vivían indios salvajes (...). Mi primera condena judicial fue en el año 98, estuve algunos meses en un instituto de menores, ahí fue donde comencé a leer revistas de todo tipo: Noticias, Pronto, Hombres, Paparazzi... Obviamente creía todo lo que leía sin cuestionar nada, todo lo daba por cierto solo porque lo escribía un periodista. También empecé a escuchar radio, pero solo programas donde pasaban cumbia colombiana o música pop que era lo que me gustaba. (Jhony)

En ese ejercicio de recorrer la historia personal para dar cuenta de los consumos, en esta práctica de distanciamiento, es posible identificar trayectorias familiares, cercanía con los afectos y con el barrio de pertenencia, pero también la puesta en valor de la educación superior en el contexto de encierro punitivo y el poder pensar la universidad como un horizonte de posibilidades.

Problematizamos los discursos mediáticos que reproducen relaciones de dominación a partir del miedo al delito (la inseguridad) que, parafraseando a Rossana Reguillo (2000), se experimenta individualmente, pero se construye socialmente y se comparte culturalmente. Indagamos sobre las coberturas que nos atraviesan y que se disputan cotidianamente desde la práctica (como estudiantes en situación de privación de la libertad, como docentes, como 
manifestantes en una marcha, como militantes de un sindicato o una organización, como mujeres, como migrantes).

En este sentido, en cada clase se propuso la reflexión acerca de los modos en los que los medios de comunicación construyen la información y su relación con los intereses propios; pensando también desde el lugar de consumo para poder conceptualizar esos usos y apropiaciones. ¿Qué emociones nos producen esas coberturas? ¿Qué sensaciones, impresiones nos producen determinadas fotografías y títulos? También propusimos la reflexión en torno a la cobertura mediática sobre la situación carcelaria, en un contexto donde las cárceles fueron foco de una intensa campaña mediática contra una supuesta liberación masiva de personas privadas de la libertad para descomprimir la sobrepoblación en espacios que constituyen un riesgo para la circulación del virus COVID-19.

Me pregunto por qué no plasman por los canales de televisión todas estas cosas: familias de personas en contexto de encierro que esperan más de tres horas para poder visitar a sus familiares, bajo lluvia, frío; agentes del SPB que abusan del pequeño poder coercitivo que tienen y les roban a las familias las cosas que traen para compartir con la visita; jueces que siguen teniendo detenidas a personas que están pasadas de todos sus beneficios y en término para poder otorgarles la libertad (Andrés).

Considerando que es necesario ahondar en estas cuestiones, reflejarlas, para que el Estado pueda mejorar las instituciones carcelarias. Así, todas y todos los privados de libertad podrán transitar el camino de reeducación y resocialización. Los derechos de las personas privadas de libertad, son siempre vulnerados. Estas incidencias, no son las más reflejadas en las estadísticas de los organismos de derechos humanos y de tribunales superiores. (Gerardo) 
En el día a día en una cárcel el porcentaje de procesados refleja un sistema corrupto que no avanza para mejor, sino que retrocede y va en aumento. Imagínense estar privado de su libertad siendo inocente y el Estado tarde cuatro años en comprobar dicha inocencia... (Ignacio)

A su vez, la impronta de recuperar sus trayectorias y vivencias específicas como estudiantes privados y privadas de la libertad también estuvo presente en el momento del examen final integrador, a través del cual buscamos plantear ejercicios de reflexión conceptual, de análisis (noticias, viñetas) y de producción (elaborar una nota de opinión o diseñar la tapa de un diario con temas prioritarios). Propusimos interrogantes acerca de los contenidos abordados en la bibliografía propuesta durante la cursada, así como también priorizamos los problemas cotidianos de las cárceles y cuáles de ellos serían noticiables para los medios de comunicación, proponiendo reflexiones acerca de lo noticiable y la toma de posición al respecto.

Mediante esta estrategia de evaluación, intentamos que fuera una instancia formativa más, a partir del desarrollo de dichas consignas. En el ciclo lectivo 2020, previo a esta instancia, tuvimos una clase de consulta, a demanda del grupo de estudiantes, que realizamos a través de la plataforma Zoom, para despejar dudas y clarificar la modalidad y criterios de la evaluación.

El esperado encuentro se hacía necesario, tanto para docentes como para el grupo de estudiantes. Allí pudimos ver sus rostros y pudimos encontrarnos con los nuestros. Nosotras, sin querer, nos metimos en su cotidiano. En esos espacios habitan, celdas o pabellones colectivos, en cárceles de máxima seguridad del complejo carcelario de La Plata. Lugares oscuros, apretados, húmedos y hacinados. 
Allí estaban Janeth, desde la Unidad 33 de mujeres Los Hornos (que no pudo continuar la materia por limitaciones de su celular y, en consecuencia, prioriza preparar materias libres con el material en papel que dispone); Marce, Rodrigo y Juan compartiendo celda en la U9; Pablo en otro sector de la misma unidad; Maxi en la U1 Olmos; Jonathan apareció también de fondo. Durante la conversación afloraron demandas que exceden los temas de la materia (como nos pasa también la presencialidad), pero que pudimos escuchar y canalizar institucionalmente. Demandas vinculadas, principalmente, a las limitaciones respecto de la conectividad y a la comunicación con las autoridades estatales.

¿Cómo garantizar la presencia política y simbólica de la Universidad en este contexto? Durante el ASPO los equipos de la modalidad educativa en contexto de encierro de la Facultad, de la Universidad y de diversas agrupaciones estudiantiles se acercaron a los establecimientos penitenciarios a alcanzar donaciones de elementos de bioseguridad, de higiene, así como también material de estudio. En tanto la UNLP dispuso, de cara al segundo cuatrimestre, módems de emergencia, en las computadoras que se encuentran disponibles en espacios colectivos de los centros de estudiantes, para facilitar y garantizar una mínima conectividad (a través del Programa de Acompañamiento Universitario en Cárceles (PAUC), Presidencia UNLP.

\section{A pesar de la virtualización}

La emergencia sanitaria expuso no sólo la crisis habitacional de las cárceles, sino también las brechas digitales en este contexto: la problemática de la conectividad, el derecho a la comunicación y a la información se muestra como una de las claves para la inclusión educativa. Un desafío en este nuevo escenario será considerar la presencia de la conectividad, sin perder de vista la posición política mantenida por la unidad académica de sostener una pedagogía de la presencia en esta modalidad educativa específica.

A pesar de la virtualización de la propuesta pedagógica de la cátedra (y de otros espacios curriculares que se dictaron en modalidad en línea), estuvo no sólo la materia, estuvieron no sólo los equipos docentes procurando el encuentro con las y los estudiantes, estuvo -una vez 
más- la presencia material y simbólica de la Universidad Pública en la cárcel; en una coyuntura donde las actividades educativas y culturales, además de las visitas de familiares y operadores judiciales en contextos de encierro fueron interrumpidas por la pandemia.

En este sentido, la perspectiva de la educación y de la comunicación asumidas como derechos marca una clara diferencia en territorios donde históricamente suelen ser administrados como beneficios.

\section{Referencias bibliográficas}

AA.VV. (2020). "Programa 2019. Modernidades, medios y Poder. Cat II". La Plata: FPyCS-UNLP.

AA.VV. (2020) Web de cátedra: https://perio.unlp.edu.ar/catedras/modernidadescat2/. La Plata: FPyCS-UNLP.

Bracchi, C. (2004). Los "recién llegados" y el intento para convertirse en "herederos": un estudio socioeducativo sobre estudiantes universitarios. Buenos Aires: FLACSO.

Bracchi, C. (2016). "Descifrando el oficio de ser estudiantes universitarios: entre la desigualdad, la fragmentación y las trayectorias educativas diversificadas" en Trayectorias Universitarias, 2, La Plata: UNLP.

Reguillo Cruz, R. (2000). Emergencia de culturas juveniles. Estrategias del desencanto. Buenos Aires: Ed. Norma. 